\title{
Study on the City Color Planning Strategy Based on the Historical Context Inheritance - Take Bailu Town, Pengzhou City, Sichuan Prov. China for Example
}

\author{
Xiaomeng Lou, Ying Meng \\ School of Architecture and Urban Plannin, Southwest University for Nationalities, Chengdu, China \\ Email address: \\ 671813076@qq.com (Xiaomeng Lou),396877993@qq.com (Ying Meng) \\ To cite this article: \\ Xiaomeng Lou, Ying Meng. Study on the City Color Planning Strategy Based on the Historical Context Inheritance - Take Bailu Town, \\ Pengzhou City, Sichuan Prov. China for Example. Humanities and Social Sciences. Vol. 4, No. 4, 2016, pp. 100-105. \\ doi: 10.11648/j.hss.20160404.13
}

Received: April 10, 2016; Accepted: July 1, 2016; Published: July 7, 2016

\begin{abstract}
With the promotion of living standard, the requirements of the life environment quality also continues to increase, but city presents the color problems go against the requirements and has affected the life quality. City color is an important part of the living environment and the carrier of the city's historical context. The scientific methods and Strategies of city color planning will play an important role in city construction and environmental regulation. In this paper, through the field research of the color in Bailu town and the color extraction and summary of Its natural environment, historical buildings, public service facilities, marking systems, advertising signs, ground pavement, we have put forward the future city color palette of Bailu in elegant gray and light color, created a "elegant gray light make-up at White Hart" color landscape, demonstrated the planning target of Chinese and Western town charm. And we have also make the district color planning control in Bailu color landscape, Trying to create the visual experience of the Western Sichuan, and a small town that can talk with color. In this paper, we explore the city color planning strategy of Bailu town based on the local cultural heritage content, combined with the actual case which Enriched the local cultural heritage content and connotation of the city, Emphasized the importance of local culture inheritance in color, Perfect the relevant content of city design. Through in-depth analysis of the inheritance and development of local culture in the case of city color planning in Bailu town in specific, to provide a practical reference for the color planning of small cities and towns with traditional cultural charm, and also provide a certain foundation and platform for the study of the city color planning of small cities and towns from the perspective of local culture in the future.
\end{abstract}

Keywords: City Color Planning, Historical Context, Inheritance, Strategy

\section{Introduction}

\subsection{Description of Study Area}

Bailu town is located in the north of Pengzhou City, the mountainous area of the northern edge of the Chengdu Plain, 38 kilometers from the city of Pengzhou, and $78 \mathrm{~km}$ from the city area of Chengdu. Bailu town is located in the Western Sichuan tourism line, with the completion of provincial forest park-Bailu forest park construction, Bailu town with Pengzhou City and the outside world will contact be more closely, and it become more and more important for the social economic status of Pengzhou City. After the " $5 \cdot 12$ " earthquake, combined the opportunity of post disaster reconstruction and the original and overall planning, they relied on the natural and historical cultural resources of Bailu town, and created the Sino French traditional customs town (Figure 1).

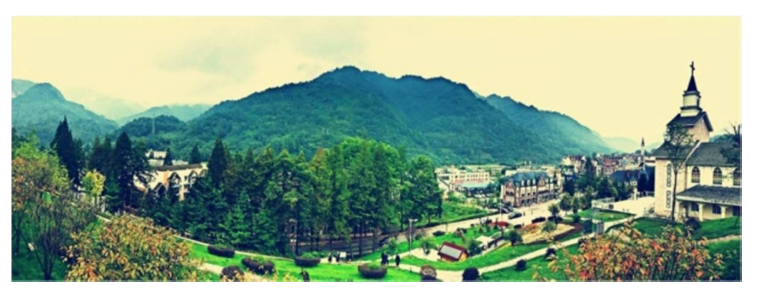

Figure 1. Panoramic view.

\subsection{Current Situation of Research on City Color}

Related to city color, the relevant scholars have done some 
research. Zhongqiang Wang (2013). To analyze the color of Kunming, he used the methods like image acquisition, color analysis, classification. He had analyzed the Allowable color within range between the tone color and tone color hue to solve and raise quality of human settlements environment. In the same time, they had built a visual image of Kunming to Increase the psychological identity of city residents to the living environment and Promote the economic development of the city and strengthening the construction of spiritual civilization in the city. Through the analysis of the city texture and city color planning of the main city of Kunming, the author had sum up the Kunming City heritage building, Central Business District, office buildings, residential areas, concentrated green, typical street along the street facade of the color image survey and research and Kunming during the cityization process and current development mode. He had analyzed city color planning of Kunming Comprehensively, and from a multi angle, combined with the specific case and analyzed the main city color planning of Kunming to achieve the overall planning of the city in the direction of the controllability. Yueyi Wang, Zhu Wang, Yong He (2013) By studying the color planning cases in two typical cities Jaipur in India and Hangzhou in China, and making analysis and comparison in the city color planning measures and results to find out their own characteristics, they had get three strategies to improve the city color planning are obtained: to create and implement the color planning and transformation, and to protect and renew the traditional regional color. Guanghua Sun, Jiangang Xu, Wei Li, Ming Li (2014). On the basis of domestic city color planning, they had drawn lessons from statutory planning system and set up a set of color planning system including overall color planning, control of color planning and color rectification plan. Among them, the overall color planning is based on the analysis of status quo of city color to propose the overall positioning of city color and determine the overall tone of the city and the total chromatogram. Control of color planning is based on the overall color planning, to divide the city into a number of management partitions, and control different types of buildings in the area of color. Color rectification plan is the final implementation of the city color planning, which focus on the city area for the implementation of the implementation of the proposed color transformation. Finally, they take Luoyang city as an example, the city color planning of Luoyang city was completed within the framework of the city color planning system.

\section{Status of City Color in Bailu Town}

\subsection{Natural Color}

Natural color has composited he background color of Bailu and is an important part of the influence in Bailu color. Natural color in Bailu town include the color of vegetation, water, soil, the sky. These harmony and unity color are the background of Bailu, whether the architectural colors or facilities colors should be coordinated. It is worth noting that with the season changing, Bailu town natural color will also change. At present, through the investigation, there are colorful vegetation, fertile soil, good water quality in Bailu town, but the waterfront environment lack of rectification, and need the planning and design adjustment (Figure 2).

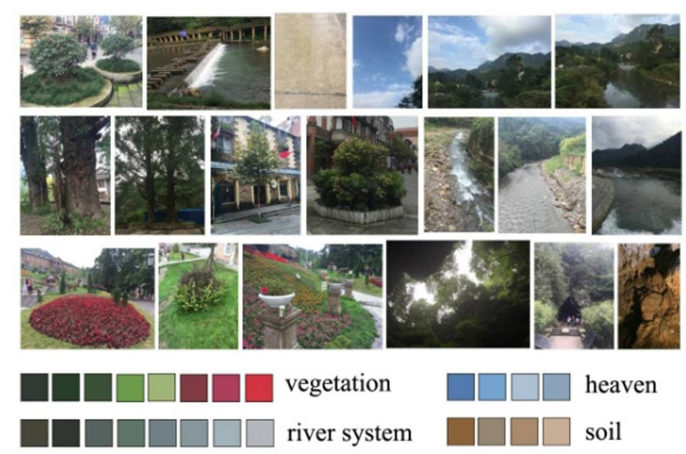

Figure 2. Natural color.

\subsection{Historic Buildings}

\subsubsection{SEMINARIUM ANNUNTIATIONIS}

SEMINARIUM ANNUNTIATIONIS called the academy, is the national key protect cultural relics, and the most important historical buildings in Bailu town. SEMINARIUM ANNUNTIATIONIS combined with a unique blend of Rome style and Gothic architectural style. It used to be a courtyard style building group with spiritual cultivation, prayer, teaching, life. The main building of SEMINARIUM ANNUNTIATIONIS has three layers, and looked very solemn and solemn. The whole building is French, including the dome, lobby and carved exterior, and looked very magnificent. The college is located in the halfway up the mountainside, from west to east. In the front, the Bailu River meandering through, and it is alongside the hill and river with excellent Feng Shui. From afar, white columns and black tiles, present the unique Chinese and Western style architectural charm of SEMINARIUM ANNUNTIATIONIS. Not only have typical of Rome style and Gothic architectural features, but also have the Chinese courtyard space characteristics.

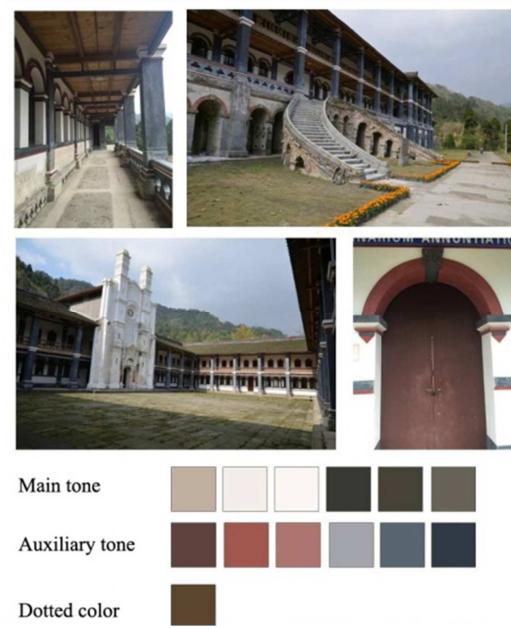

Figure 3. SEMINARIUM ANNUNTIATIONIS. 
SEMINARIUM ANNUNTIATIONIS's main colors for white and black, mainly in the color of the walls and roof of building, the secondary color is gray and scarlet and blue gray, mainly referring to the post, color of window and door frames, decorative color is brown, mainly referring to the is building decoration color. Collar repair hospital is Bailu's main historic buildings and the use of its architectural color is typical and representative, the late stage of planning should be the continuation and inheritance of the color scheme (Figure 3).

\subsubsection{Old Street}

Street Hebachang Bailu Town, is the gwhite hnart field, is one of the ten ancient town of Chengdu, first built in the period of Qian Long Kangxi. The whole street on the Bailu River built, short, long from north to south. Looking far away like a giant ship, street building most wood structure wear beam, Xiaoqing tile roof. After the earthquake, street ancient architecture is almost completely destroyed. Today, the streets of the rebuild Chuanximinju building on the site, architectural style.

Through on-site investigation and mapping, street roof and wall color constitute the basic distribution of the color main tone of in the blue gray range, the secondary color is the color of the door frame and window, mainly wood color and dark red, decorative color is beige, architectural decoration color (Figure 4).

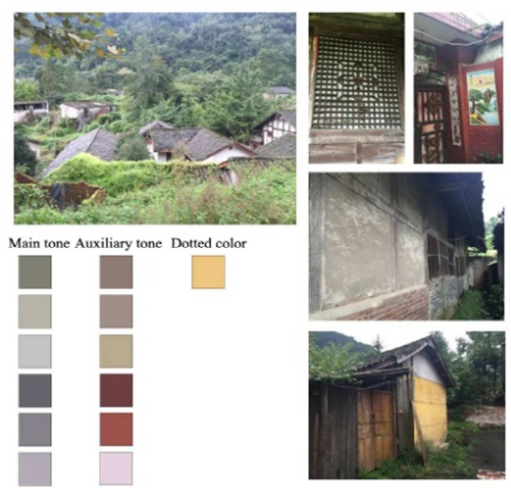

Figure 4. Old Street.

\subsubsection{Most Cattle Teaching Building}

The teaching building is in the May 12 earthquake, Bailu town center school buildings basically are in the earthquake collapsed, except the two building buildings stand down, the teaching building of the cattle and it in front of the building, originally is in the same horizontal plane, after the earthquake, on the mountain of the teaching building due to the interaction of fault, to uplift the 3 meters, but the stairs are still robust, was only slightly damaged, and more than 1000 teachers and students in the school are safe evacuation. Most cattle teaching building belongs to modern architecture, architectural color is white and gray, because of its higher historical commemorative value, so planning should unchanged (Figure 5).
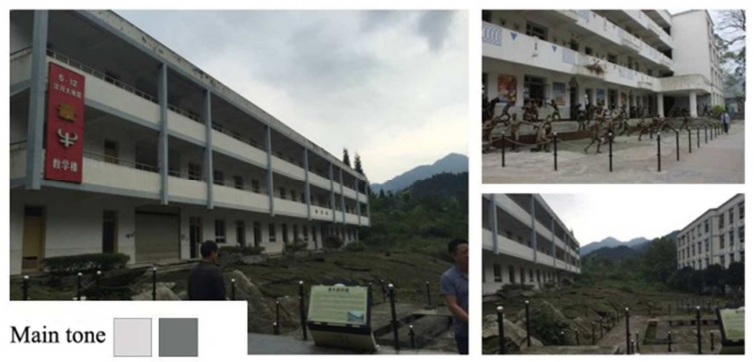

Figure 5. Most cattle teaching building.

\subsubsection{Sino French Bridge}

French bridge, also known as Jinqiao, was founded in 1893 , is located in the town of Jinqiao village 100 meters northwest of white deer. East-west, masonry structure, double arch bridge, east of three white road, West to the Academy gate is when leading the path for the Academy. Sino French bridge deck with green stone paved, only a single hole after the earthquake, the bridge has become a historical relic. Sino French bridge's main color is green gray, because it is the important history of the building, therefore planning design should retain the original appearance (Figure 6).
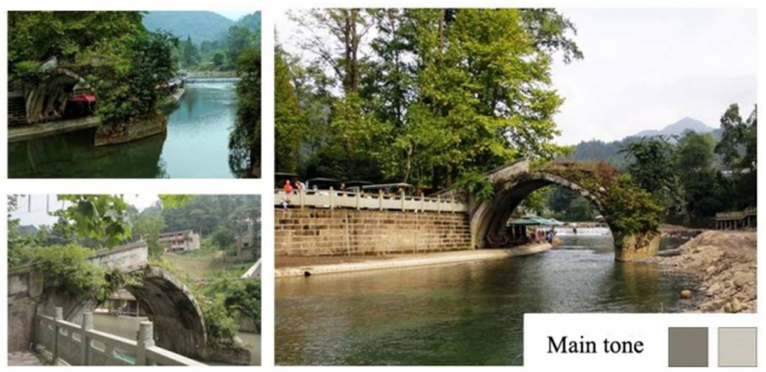

Figure 6. Sino French Bridge.

\subsubsection{Public Service Facilities}

Bailu's public facilities of color, mostly using color saturation range difference, presents an overall color trend compared with coordinated, but there are also other facilities appear to conflict with the overall tone of the color, the late stage of planning should remediation, the coordination and unity (Figure 7).

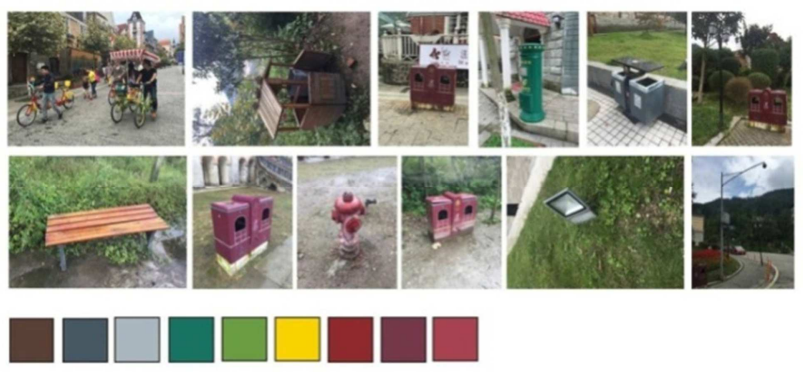

Figure 7. Public service facilities.

\subsubsection{Identification System}

Color of Bailu identification system has two color tendency, in green spaces and plants in the vicinity of the skewed to the gray green, and in the attractions in the vicinity 
of skewed to the red brick, the use of the color give full consideration to the coordinated with the surrounding environment, worth preserving and reference, but there are few marking system and the two tone is not uniform, the latter should remediation (Figure 8).

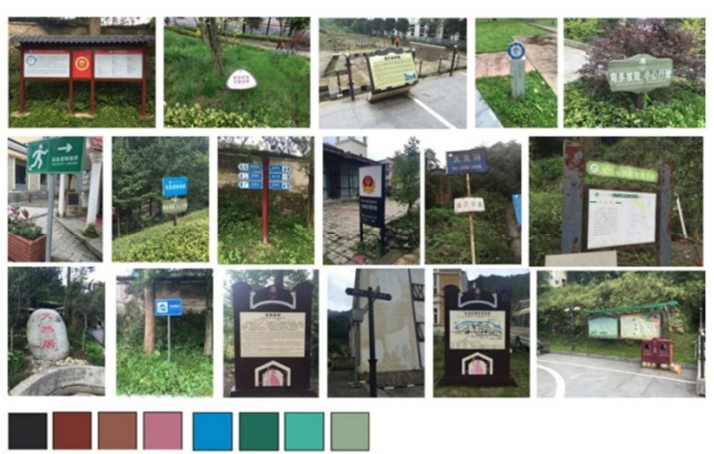

Figure 8. Identification system.

\subsubsection{Advertising Signs}

Bailu town advertising signs rarely use very unexpected, high purity of color, but the tone of the unity with the surrounding buildings, so it looks like the whole street did not because of the influence of billboards and become disorganized, but rather for street hyperchromic many. But there are still some temporary advertising signs and the surrounding color is not coordinated place, should be appropriate to control the emergence of such a situation, so as not to affect the street appearance (Figure 9).

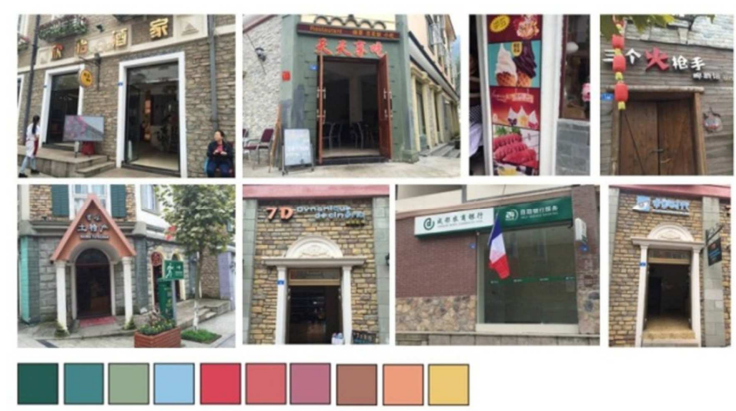

Figure 9. Advertising signs.

\subsubsection{Floor Covering}

Bailu of pavement materials and rich and varied forms, but the color is controlled in a comfortable range, gray tone, the use of a good background of the architectural beauty (Figure 10).

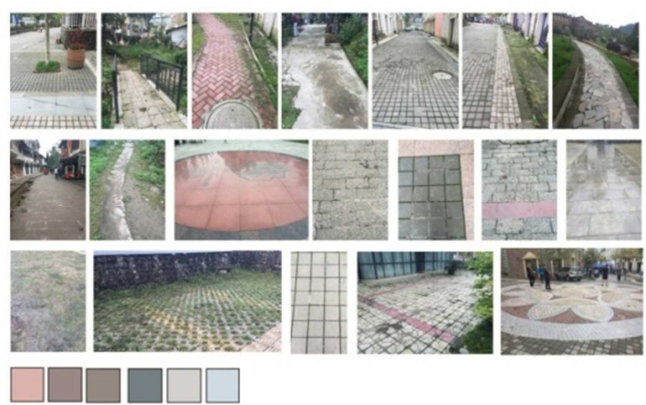

Figure 10. Floor covering.

\section{Bailu Town City Color Planning Strategy}

\subsection{Planning Objectives}

Present comprehensive research and the town of Bailu future development, Bailu town in the future the main colors planning positioning for "elegant grey, light color tone. The Bailu Township color planning objectives, create a "elegant pale gray color decorative White Hart" color landscape, highlighting the Chinese and western town charm. Color planning look elegant and bright, base color, construction Wailimian color wall main color to light gray color roof to the clever degree, low brilliant color.

The inheritance of historical and cultural elegance grey (Figure 11) and the use of historical buildings of the town of Bailu elegance grey tone inheritance, Chinese architecture and French architecture with tonal and unified, inheritance historical context. Decorative White Hart future light color (Figure 12), light color use like a beautiful woman makeup, always as a voracious reader and full of flavor. In terms of the type of tourism in the town of Bailu, clever degree, low Yan of color makes the city more than a vibrant atmosphere and as for form vision of conflict with a variety of tourists is meet all directions to visitors the best color state.

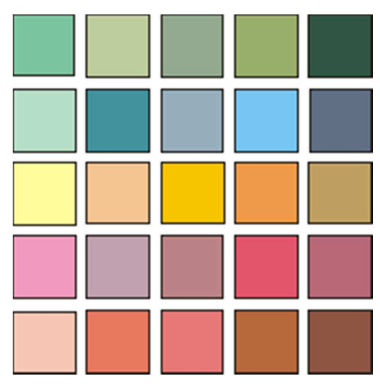

Figure 11. Elegance grey.

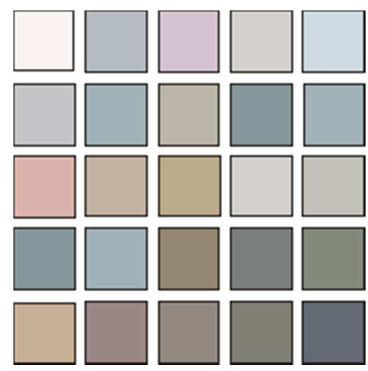

Figure 12. Light color.

\subsection{Color Zoning Control Planning}

Bailu town color layout to form the overall layout of a shaft, two hearts, three kilometer (Figure 13). "Axis" refers to the Bailu River along the connection Sanhe shop, the Annunciation school, towns, Bailu Village and other important attractions of city color landscape axis. "Two hearts" refers to the center of the town and the Libao monastery based cultural tourism center. "Three area" refers to the center of the town and the Annunciation convent 
region division of color control area and the surrounding area to form two pieces of color harmony.

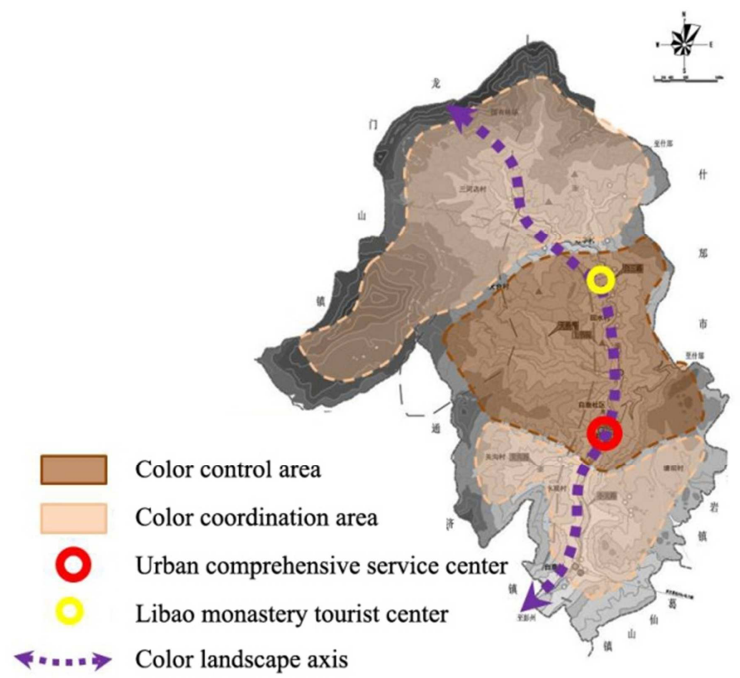

Figure 13. Color zoning control planning.

Color control area is recommended to use color: color control district is the center of the town and the Annunciation college is located in the region, town of Bailu color focus and climax, building facilities and the surrounding colors should be coordinated with the surrounding environment, and controlinless than recommended color (Figure 14).

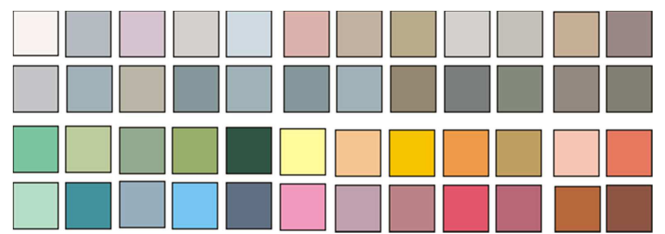

Figure 14. Color control area is recommended to use color.

Recommended use of color for color coordination area: color coordination area of the use of color should be by the beauty of the whole city, foil as a guide, pursuit of city overall color of harmony and unity, color lightness, purity and chroma should try to control within the recommended color (Figure 15).

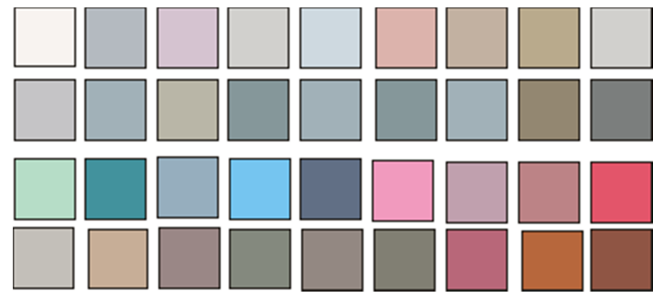

Figure 15. Recommended use of color for color coordination area.

\section{Epilogue}

City color is an important part of the city living environment quality and an important carrier of city history and culture, and also the embodiment of modern civilization.
Therefore, the city color design of scientific methods and research will play an important role in city construction and environmental remediation, and it is particularly important through city color planning to protect and display regional culture and historical. Study on city color science can effectively prevent the city "color pollution", to create a livable environment for the residents. The content of city color planning mainly includes preliminary research, investigation and study of the present situation, color planning and management research, through the comprehensive investigation and study of planning object natural environment, historic buildings, buildings and other aspects. Through scientifically analyzed and summarize, we develop a suitable scheme of city color planning, extractthe main tone of city characteristics, induce city chromatography, and then make the management departments to facilitate control and the final implementation. In this paper, through the field research of the color in Bailu town and the color extraction and summary of Its natural environment, historical buildings, public service facilities, marking systems, advertising signs, ground pavement, we have put forward the future city color palette of Bailu in elegant gray and light color, created a "elegant gray light make-up at "White Hart" color landscape, demonstrated the planning target of Chinese and Western town charm. And we have also make the district color planning control in Bailu color landscape, Trying to create the visual experience of the Western Sichuan, and a small town that can talk with color. In this paper, we explore the city color planning strategy of Bailu town based on the local cultural heritage content, combined with the actual case which enriched the local cultural heritage content and connotation of the city, emphasized the importance of local culture inheritance in color, perfect the relevant content of city design. Through in-depth analysis of the inheritance and development of local culture in the case of city color planning in Bailu town in specific, to provide a practical reference for the color planning of small cities and towns with traditional cultural charm, and also provide a certain foundation and platform for the study of the city color planning of small cities and towns from the perspective of local culture in the future. Through the exploration of the methods and Strategies in Bailu town's city color planning, the author provides a reference for the city color planning, and tries to appeal to more scholars to pay attention to and participate in the city color planning ranks.

\section{Acknowledgements}

This project is supported by the innovation project of Southwest University for Nationalities, project number: CX2015SZ011.

\section{References}

[1] Zhongqing Wang. Analysis of urban color planning in the main city of Kunming [D]. Kunming University of Science and Technology, 2013. 
[2] Yueyi wang, Zhu Wang, Yong He. A comparative study on the strategies and methods of color planning in typical cities of China and India - take Jaipur in India and Hangzhou in China as an example [J]. City planning, 2013, 2013, No. 31410: 20-26.

[3] Guanghua Sun, Jiangang Xu, Wei Li, Ming Li. Construction of urban color planning system based on statutory planning system--take the color planning of luoyang as an example [J]. Planner, 2014, v. 30; No. 22711: 36-41.

[4] Meng Fu. Study on the color problem in Harbin city construction [J]. Heilongjiang Science and Technology Information, 2016, 06: 235.

[5] Yang Xue. Preliminary study on urban color planning--take Wuhan as an example [J]. Huazhong Architecture, 2016, v. 34; No. 22603: 145-147.

[6] Yuan Gao, Baozhu Li. Study on urban architectural color planning and management-take the main city in xuzhou as an example [J]. jiangsu architecture, 2016, No. 17401: 15-19.

[7] Honglei $\mathrm{Xu}$, Jing Ren. Xi'an city color system planning and teaching research $[\mathrm{J}]$. China Education Journal, 2015, S1: 80-81.

[8] Feng Yang. The special planning of urban color and architectural style in Dalian City [J]. planner, 2015, v. 31; No. 24012: 114-121.

[9] Aiyun Yang. The use of urban color to shape the spirit of Yiyang City [J]. city, 2015, v. 36; No. 16304: 58-60.

[10] Guanhua Fu, Jing Liang. Design color and social psychology research-take city color as example [J]. Beauty and age (I), 2015, No. 63212: 49-51.

[11] Yi Liu, Min Zhang, Xiaojuan Li. On urban color planning strategy from the perspective of urban color image [J]. Art and design (Theory), 2015, v. 2; No. 31612: 61-63. 